\title{
Strong Exciton-Photon Coupling in a Nanographene Filled Microcavity
}

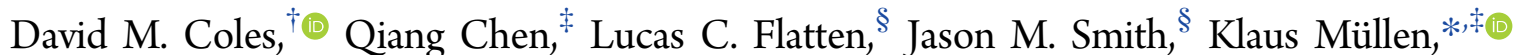

Akimitsu Narita, ${ }^{*}+\infty$ and David G. Lidzey* ${ }^{*}$

${ }^{\dagger}$ Department of Physics \& Astronomy, University of Sheffield, Sheffield S3 7RH, United Kingdom

${ }^{\ddagger}$ Max Planck Institute for Polymer Research, Mainz D-55128, Germany

${ }^{\S}$ Department of Materials, University of Oxford, Oxford OX1 3PH, United Kingdom

\section{Supporting Information}

ABSTRACT: Dibenzo[hi,st] ovalene (DBOV)—a quasi-zero-dimensional "nanographene"-displays strong, narrow, and well-defined optical-absorption transitions at room temperature. On placing a DBOV-doped polymer film into an optical microcavity, we demonstrate strong coupling of the $0 \rightarrow 0^{\prime}$ electronic transition to a confined cavity mode, with a coupling energy of $126 \mathrm{meV}$. Photoluminescence measurements indicate that the polariton population is distributed at energies approximately coincident with the emission of the $\mathrm{DBOV}$, indicating a polariton population via an optical pumping mechanism.

KEYWORDS: Microcavity, nanographene, strong coupling, polaritons

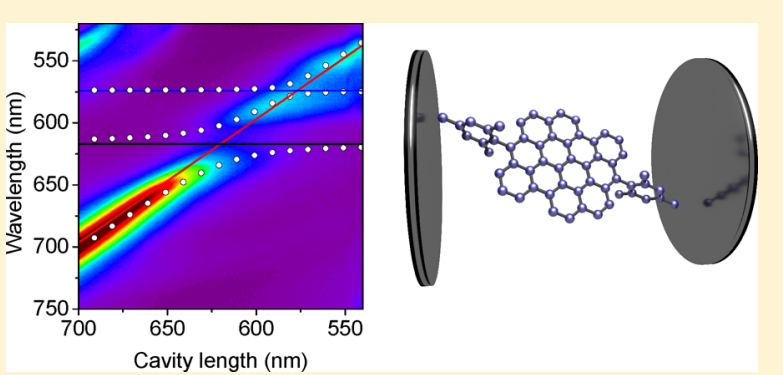

raphene has fascinating electronic properties as demon-

$\mathcal{J}$ strated by its high charge-carrier mobility; however, it does not possess a band gap, a feature that limits its applications in transistors and optical devices. ${ }^{1}$ A number of approaches have been explored to overcome this obstacle, including doping, ${ }^{2,3}$ controlled interactions with a substrate, ${ }^{4,5}$ and the application of electric fields to graphene bilayers. ${ }^{6}$ However, the structural confinement of the graphene sheet into a few nanometers appears to a promising strategy to create a band gap, with "nanographene" structures developed including quasi-one-dimensional graphene nanoribbons $(\mathrm{GNRs})^{7}$ or quasi-zero-dimensional graphene quantum dots (GQDs)., Top-down fabrication methods do not however allow the preparation of nanographenes with well-defined structures and properties, and thus we have developed a bottom-up chemical synthesis route to prepare atomically precise GNRs and GQDs. ${ }^{10,11}$ Here, key optical and electronic properties such as optical absorption and energy gaps can be efficiently controlled by modulating the size, geometry, and edge structure of the nanographenes, making them promising for (opto)electronic applications. ${ }^{12-14}$

There is significant growing interest in the development of new materials for polariton condensation; polaritons are quasiparticles that are formed in optical cavities when an optically active material reversibly exchanges energy with a resonant electromagnetic (EM) field. Here, the energy levels of the transition dipole and EM field become hybridized, resulting in new energy eigenstates that can be described as part excitation and part photon. A convenient method to reach the strong coupling regime is to place a semiconductor material into an optical microcavity. Providing that the interaction strength between the excitons and the confined electromagnetic field is dominant over their respective loss channels, the excitons can couple to the confined cavity mode to form exciton-polaritons. Because the cavity mode energy dispersion effectively forms a trap in momentum space, it is possible to build a large population of polaritons at the bottom of the trap with the bosonic polaritons undergoing condensation at a high occupation density. ${ }^{15}$ This can result in the generation of nonlinear phenomena such as inversionless lasing ${ }^{16}$ and polariton superfluidity. ${ }^{17}$

The strong coupling regime has been demonstrated with a range of different semiconductor materials and hetrerostructures, including bulk semiconductors, ${ }^{18}$ semiconductor quantum wells, ${ }^{19}$ two-dimensional transition metal dichalcogenides, ${ }^{20,21}$ and organic (molecular) dyes. ${ }^{22-24}$ More recently, single-walled carbon nanotubes have been shown to undergo strong coupling in a microcavity. ${ }^{25}$ Nevertheless, to the best of our knowledge, such strong coupling has never been observed in graphene-based materials. Graphene in optical cavities has been previously studied; ${ }^{26,27}$ however, in those studies, the materials remained firmly in the weak coupling regime whereby the cavity acts to perturb the transition rates of the graphene (the "Purcell effect"), rather than create entirely new polariton states. To explore whether nanographene materials can reach the strong coupling regime, we have explored the application of

Received: May 25, 2017

Revised: August 15, 2017

Published: August 22, 2017 
a new nanographene material (dibenzo[hi,st $]$ ovalene $[\mathrm{DBOV}]$ ) in a microcavity. DBOV is particularly interesting for this application as it combines high oscillator strength and wellresolved electronic and vibrational transitions. ${ }^{28}$ It also displays remarkable photostability $^{28-30}$ compared to other organic materials used for microcavity strong coupling ${ }^{31-35}$ (see Supporting Information) and is able to undergo amplified spontaneous emission (ASE). ${ }^{28}$ We place this material in an open-cavity architecture and demonstrate that strong coupling occurs to the electronic state of DBOV, with polaritonic emission evidenced at room temperature. We believe that the relatively high quantum efficiency of luminescence and high chemical stability of such materials make them promising candidates for polariton condensation and use in nonlinear polariton-based devices.

The DBOV derivative we have explored (DBOV-Mes) has been synthesized though a bottom-up chemical process (see SI for details). The chemical structure of DBOV-Mes is shown in the inset of Figure 1a and consists of a polycyclic aromatic
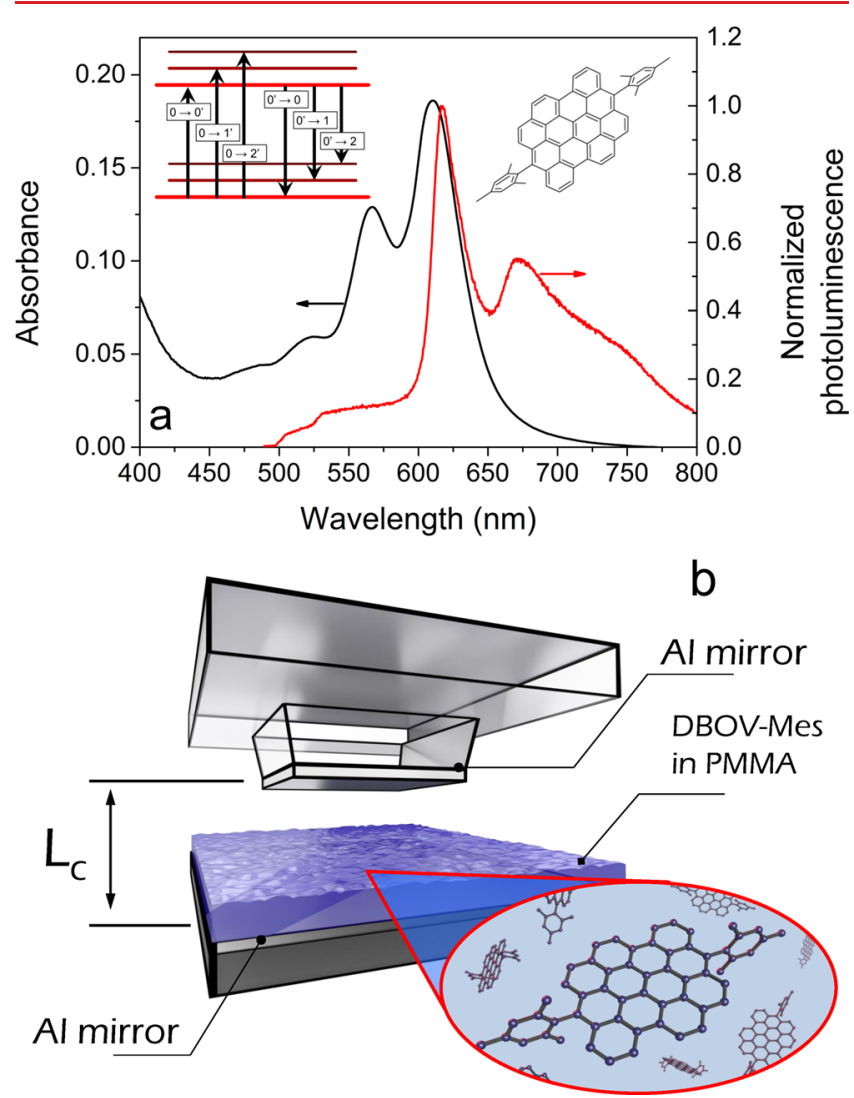

Figure 1. (a) Absorbance (black line) and photoluminescence (red line) of DBOV-Mes in a PMMA matrix. Left inset: Energy level diagram. Right inset: chemical structure of DBOV-Mes. (b) Microcavity structure consisting of a DBOV-Mes:PMMA film between two metallic mirrors with tunable separation, $L_{c}$.

hydrocarbon core with attached solubilizing mesityl groups that also provide kinetic protection to the relatively reactive zigzag edges. To process DBOV-Mes, it was dissolved in a solution of poly(methyl methacrylate) (PMMA) in dichloromethane (15 $\mathrm{mg} / \mathrm{mL}, 1: 1$ DBOV-Mes:PMMA weight ratio) and then spincast to form a $540 \mathrm{~nm}$ thick film. The absorbance of such a film is shown in Figure 1a (black line). Here, it can be seen that there is a strong peak in absorbance at $610 \mathrm{~nm}$ having a full- width at half-maximum (fwhm) line width of $41 \mathrm{~nm}(135 \mathrm{meV})$ corresponding to the $0 \rightarrow 0^{\prime}$ electronic transition, with weaker vibronic replicas at $565 \mathrm{~nm}\left(0 \rightarrow 1^{\prime}\right)$ with a fwhm of $36 \mathrm{~nm}$ $(139 \mathrm{meV})$ and $518 \mathrm{~nm}\left(0 \rightarrow 2^{\prime}\right)$ with a fwhm of $48 \mathrm{~nm}(220$ $\mathrm{meV})$. Following excitation at $405 \mathrm{~nm}$, the film emits strong photoluminescence as plotted using a red line. Here, the $0^{\prime} \rightarrow 0$ transition is prominent, having a Stokes shift of $<10 \mathrm{~nm}$. Emission from the $0^{\prime} \rightarrow 1$ transition is also evident, while the emission from lower energy transitions is present but not wellresolved. We believe that the relatively narrow line width of the optical and vibrational transitions and small Stokes shift point to a highly rigid molecular structure, which results in a low degree of conformationally induced broadening. Furthermore, the DBOV-Mes sample used here consists of GQDs having a single, atomically defined structure: a feature that prohibits inhomogeneous broadening typical in top-down-fabricated GQDs that consist of a complex mixture of undefined structures. Photoluminescence observed below $600 \mathrm{~nm}$ is believed to be due to a tiny amount of the benzo[ $a]$ dinaphtho$\left[2,1,8-c d e: 1^{\prime}, 2^{\prime}, 3^{\prime}, 4^{\prime}\right.$-ghi] perylene derivative, which was derived from the precursors and was present in the final sample (see the SI).

To observe strong coupling, the GQD/PMMA film was deposited by spin-coating on a $20 \mathrm{~nm}$ thick semitransparent aluminum mirror. A second mirror that had been deposited onto a small raised plinth $(\sim 100 \mu \mathrm{m}$ square $)$ was brought into close proximity $(<1 \mu \mathrm{m})$ using a piezoelectric actuator, giving precise control over the mirror separation through application of a voltage. This forms an open cavity configuration as shown in Figure 1b. Here, the use of a plinth facilitates the close positioning of the mirrors without problems resulting from the presence of unwanted dust or surface contamination which might otherwise prevent the mirrors from "closing". To study the optical properties of the cavity, white light was focused onto the cavity, with the transmitted light imaged onto the entrance slit of an imaging CCD spectrometer using an infinity-corrected long working distance objective lens.

The cavity path length, $L_{\mathcal{c}}$ was first reduced to the order of a few microns. At this point, well-defined Fabry-Perot peaks become visible in the transmission spectrum having wavelengths $\lambda_{q}=\frac{2 n L_{c}}{q}$ where $q$ is the mode number and $n$ is the intracavity refractive index. The mode number for each peak can be identified from its wavelength and the wavelength of the adjacent mode on the low energy side via $q=\frac{\lambda_{q-1}}{\lambda_{q-1}-\lambda_{q}}$. The transmission through the cavity as a function of wavelength and cavity length is shown in Figure 2a. Here, a peak in transmission is observed at $\sim 700 \mathrm{~nm}$ for a cavity length of $700 \mathrm{~nm}$ corresponding to the $q=3$ cavity mode. The line width of this peak is $19 \mathrm{~nm}(49 \mathrm{meV})$, corresponding to a $Q$ factor of $\sim 35$. As the cavity length is further reduced, the mode energy increases and approaches the $0 \rightarrow 0^{\prime}$ transition of the DBOVMes. At a cavity length of $620 \mathrm{~nm}$, this mode splits forming polariton branches that anticross about the $0 \rightarrow 0^{\prime}$ transition energy, a characteristic signature of strong coupling. As the mirror separation is further reduced, the mode energy increases and again splits at a cavity length of $580 \mathrm{~nm}$ as it undergoes anticrossing about the $0 \rightarrow 1^{\prime}$ transition. The peak positions are shown as white circles. For completeness, Figure $2 \mathrm{~b}$ plots transmission spectra recorded from the cavity as it is closed at length intervals of $20 \mathrm{~nm}$. Here the splitting of the peaks as they 

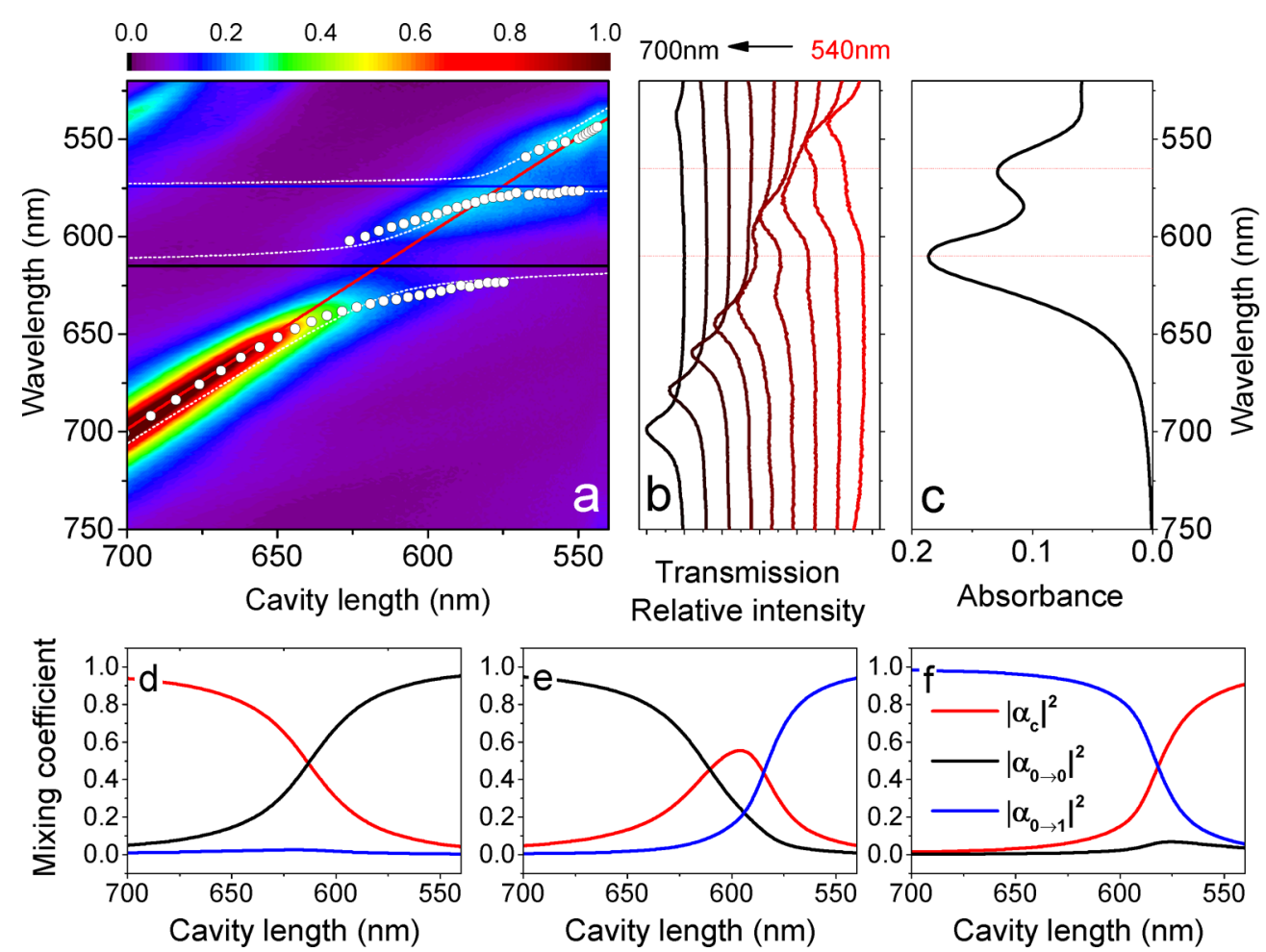

Figure 2. (a) Cavity transmission spectra as a function of optical cavity length, $L_{c^{*}}$ Open symbols are observed polariton branch wavelengths. Black and blue lines are uncoupled exciton wavelengths; the red line is the uncoupled photon wavelength as found from fitting the observed peak positions to the coupled oscillator model. White dashed lines are the modeled polariton branch energies. (b) Normalized cavity transmission spectra for various cavity lengths. The length difference between successive plots is $20 \mathrm{~nm}$. (c) Absorbance spectrum of a thin film of DBOV-Mes in a PMMA marix. The polariton branch mixing coefficients are shown for (d) the lower polariton branch, (e) middle polariton branch, and (f) upper polariton branch.

approach the electronic and vibrational resonances of DBOVMes is clearly visible.

Since three features are observed in the transmission spectra, this system can be described by three coupled classical oscillators as expressed by eq 1 .

$$
\begin{aligned}
& \left(\begin{array}{ccc}
E_{\mathrm{c}}-i \gamma_{\mathrm{c}} & \hbar \Omega_{0 \rightarrow 0^{\prime}} / 2 & \hbar \Omega_{0 \rightarrow 1^{\prime}} / 2 \\
\hbar \Omega_{0 \rightarrow 0^{\prime}} / 2 & E_{0 \rightarrow 0^{\prime}}-i \gamma_{0 \rightarrow 0^{\prime}} & 0 \\
\hbar \Omega_{0 \rightarrow 1^{\prime}} / 2 & 0 & E_{0 \rightarrow 1^{\prime}}-i \gamma_{0 \rightarrow 1^{\prime}}
\end{array}\right)\left(\begin{array}{c}
\alpha_{\mathrm{c}} \\
\alpha_{0 \rightarrow 0^{\prime}} \\
\alpha_{0 \rightarrow 1^{\prime}}
\end{array}\right) \\
& =E_{\mathrm{p}}\left(\begin{array}{c}
\alpha_{c} \\
\alpha_{0 \rightarrow 0^{\prime}} \\
\alpha_{0 \rightarrow 1^{\prime}}
\end{array}\right)
\end{aligned}
$$

Here $E_{\mathrm{c}}$ is the uncoupled cavity mode energy; $E_{0 \rightarrow x}$ is the energy of the $x$ th vibronic transition $\left(x=0^{\prime}\right.$ or $\left.1^{\prime}\right)$, and $\hbar \Omega_{0 \rightarrow x}$ is the magnitude of the energy splitting about the $x$ th transition, known as the Rabi splitting energy. The cavity mode and exciton transition line widths $\left(\gamma_{c}\right.$ and $\gamma_{0 \rightarrow x}$, respectively) are included as imaginary components on the Hamiltonian diagonal. ${ }^{36}$ The resultant eigenvalues correspond to the energy of the different polariton states created and are denoted by $E_{\mathrm{p}}$. The eigenvectors defined through the coefficients $\alpha_{\mathrm{c}}$ and $\alpha_{0 \rightarrow x}$ describe the relative fraction of photon and $x$ th vibrational transition that is mixed into each polariton. We can fit eq 1 to the experimentally observed peak positions to yield values for the Rabi splitting energies and the mixing coefficients. There are three solutions for $E_{\mathrm{p}}$ indicating that the polariton states are distributed over three branches. The lower polariton branch
(LPB) resides at energies lower than the $\hbar \Omega_{0 \rightarrow 0^{\prime}}$ transition, and the middle polariton branch (MPB) lies between the $\hbar \Omega_{0 \rightarrow 0^{\prime}}$ and $\hbar \Omega_{0 \rightarrow 1^{\prime}}$ transition energies, while the upper polariton branch (UPB) is found at energies higher than the $\hbar \Omega_{0 \rightarrow 1^{\prime}}$ transition. The fitted polariton branch positions are shown as white dashed lines in Figure 2a, along with the optical transition energies and cavity mode energy (black/blue lines and red line, respectively). The fitted energy of the $\hbar \Omega_{0 \rightarrow 1^{\prime}}$ transition is redshifted from the maximum for that transition by $\sim 10 \mathrm{~nm}$ which has attributed to residual oscillator strength on the low energy side of that transition. ${ }^{33,37}$ The coupled branch energies are plotted as dashed white lines. We find that the Rabi splitting energy about the electronic $\left(0 \rightarrow 0^{\prime}\right)$ transition is $\hbar \Omega_{0 \rightarrow 0^{\prime}}=126$ $\mathrm{meV}$, while the splitting about the first vibronic replica $(0 \rightarrow$ $\left.1^{\prime}\right)$ is $\hbar \Omega_{0 \rightarrow 1^{\prime}}=90 \mathrm{meV}$. Figure $2 \mathrm{c}$ shows the absorbance of DBOV-Mes relative to the polariton anticrossings. The definition of strong coupling requires that the sum of the cavity mode and transition half-width-at-half-maximum (HWHM) be less than the Rabi splitting energy. ${ }^{38}$ This criteria is apparently fulfilled for the coupling about the $0 \rightarrow 0^{\prime}$ transition; however, it is not quite reached for the $0 \rightarrow 1^{\prime}$ transition. Instead, coupling to the $0 \rightarrow 1^{\prime}$ transition falls into the intermediate coupling regime, ${ }^{39}$ where the coupling energy is less than the sum of the oscillator HWHMs but greater than the absolute difference of the HWHMs. This makes the definition of a middle polariton branch somewhat ambiguous; however, since nonlinear behavior in microcavity polariton systems is mainly observed in the LPB,$^{15,40,41}$ this should not effect the practical implementation of such a system. For simplicity, in Figure 2, we refer to the peak observed between 


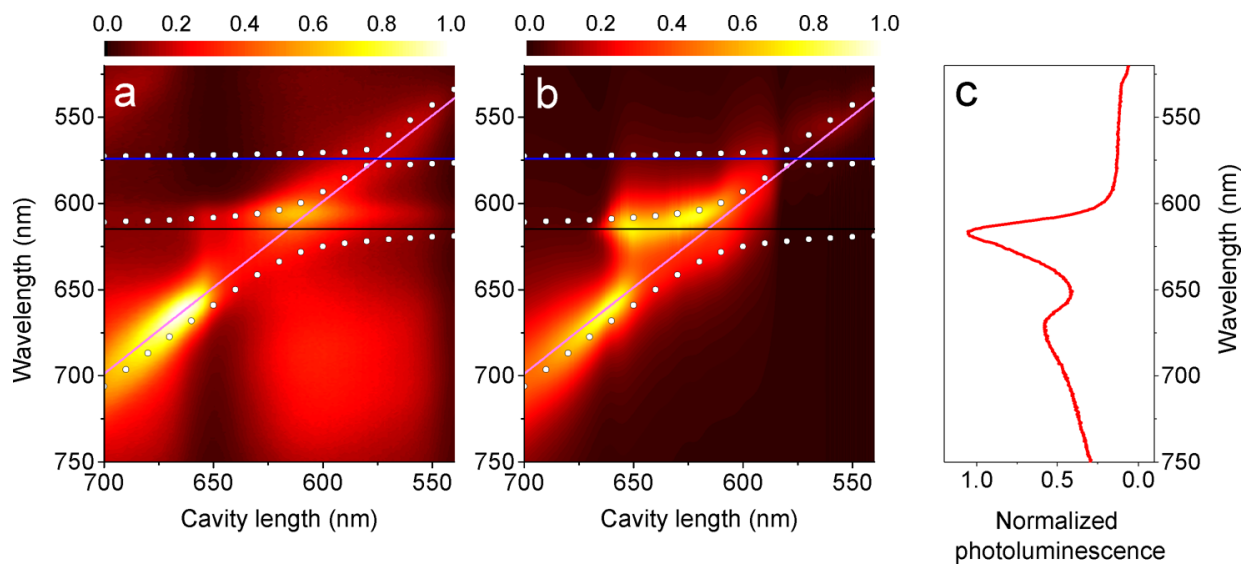

Figure 3. (a) Microcavity photoluminescence as a function of optical cavity length. Lines and symbols are as in Figure 2a. (b) Polariton population distribution. (c) PL spectrum of a thin film of DBOV-Mes in a PMMA matrix.

the UPB and LPB as the middle branch even though the requirement for strong coupling is not fulfilled here.

The mixing coefficients $\alpha_{\mathcal{c}} \alpha_{0 \rightarrow 0^{\prime}}$, and $\alpha_{0 \rightarrow 1^{\prime}}$ can be extracted from eq 1 and are plotted in Figure $2 \mathrm{~d}$, e, and $\mathrm{f}$ for the LPB, $M P B$, and UPB, respectively. We find that the LPB is photonlike at longer cavity lengths and becomes increasingly hybridized with the $0 \rightarrow 0^{\prime}$ transition at shorter cavity lengths. The UPB is largely comprised of the $0 \rightarrow 1^{\prime}$ transition at longer cavity lengths, becoming more photon-like as the cavity length is reduced. The visibility of the polariton branches is proportional to the photonic coefficient since light couples to the polariton states through this component. This effect is particularly pronounced for the LPB, with the optical transmission increasing significantly with an increased photon fraction (see Figure 2d).

We have measured the PL emission from the cavity following excitation at $405 \mathrm{~nm}$ as shown in Figure 3a, with data plotted as a function of wavelength and cavity length. We also plot the polariton energies (as determined from transmission measurements) using circular data points. We note that the laser power admitted into the cavity is strongly modulated as the cavity length is changed and is maximized when the excitation wavelength fulfils the cavity Bragg condition. To account for this, we record the excitation power transmitted through the cavity using a photodiode as the cavity length is scanned and use this signal to normalize the PL spectra recorded at each mirror separation.

We find that intense PL is observed at wavelengths coincident with the LPB, with the emission of the LPB peaking at $660 \mathrm{~nm}$ and a second emission feature at $615 \mathrm{~nm}$. The polariton PL intensity $\left(I_{\mathrm{p}}\right)$ can be related to the polariton population of any particular state $\left(P_{\mathrm{p}}\right)$ through $P_{\mathrm{p}} \propto I_{\mathrm{p}} / \alpha_{\mathrm{c}}$. We plot the polariton population distribution along the polariton branches in Figure 3b. For comparison, we plot the free space emission of DBOV-Mes in Figure 3c. Here, it can be seen that the emission originating from the LPB around $660 \mathrm{~nm}$ corresponds to the peak of the DBOV-Mes emission originating from the $0^{\prime} \rightarrow 1$ transition. We also note that the peak of the higher energy PL emission corresponds to the wavelength of $0^{\prime} \rightarrow 0$ transition energy. This suggests that polariton states are effectively populated through an optical pumping mechanism ${ }^{42,43}$ in which weakly coupled excitons in the exciton reservoir directly populate the photonic component of polariton states that are energetically degenerate with the emission. This polariton population mechanism has been shown to dominate inelastic exciton-phonon ${ }^{33,44-46}$ or polariton-polariton scattering pathways in strongly coupled systems based on materials having a high photoluminescence quantum efficiency. ${ }^{47}$ This pumping mechanism is thought to be responsible for initiating polariton lasing in microcavities containing single anthracene crystals. ${ }^{48}$ Here the polariton population generated on the MPB results from the small Stokes shift between absorption and luminescence in this material. The peak polariton population on the LPB is 1.3 times higher than that of the $615 \mathrm{~nm}$ emission, despite the fact that the PL intensity of the $0^{\prime} \rightarrow 1$ transition is almost half that of the $0^{\prime} \rightarrow$ 0 transition. Here the $0^{\prime} \rightarrow 1$ emission transition is able to emit photons resonant with the very photon-like LPB which results in efficient polariton generation. ${ }^{49,50}$

In summary, we have fabricated open optical microcavities containing structurally well-defined nanographene (DBOVMes) quantum dots. The narrow and well-separated optical transitions characteristic of DBOV-Mes lend themselves to strong exciton-photon coupling, and we demonstrate the formation of polariton states in this system at room temperature resulting from the hybridization of the cavity mode with the $0 \rightarrow 0^{\prime}$ optical transition. Intermediate coupling is also observed between the cavity and the $0 \rightarrow 1^{\prime}$ vibronic transition. Photoluminescence measurements demonstrate that coupled states are efficiently populated through an optical pumping mechanism. Strong photoluminescence is observed from states close to the $0^{\prime} \rightarrow 0$ energy as a result of the small Stokes shift and high quantum yield of this transition. Moreover, the high fluorescence quantum efficiency of DBOV-Mes (with values reported up to 0.79$)^{28}$ and remarkable photostability suggests that such nanographene materials may be suitable for generating polariton condensation and lasing.

\section{ASSOCIATED CONTENT}

\section{Supporting Information}

The Supporting Information is available free of charge on the ACS Publications website at DOI: 10.1021/acs.nanolett.7b02211.

Synthesis methods, NMR and MS spectra, absorption and photoluminescence in solution, comparative photostability measurements (PDF) 


\section{AUTHOR INFORMATION}

\section{Corresponding Authors}

*E-mail: muellen@mpip-mainz.mpg.de.

*E-mail: narita@mpip-mainz.mpg.de.

*E-mail: d.g.lidzey@sheffield.ac.uk.

\section{ORCID}

David M. Coles: 0000-0003-4808-6395

Klaus Müllen: 0000-0001-6630-8786

Akimitsu Narita: 0000-0002-3625-522X

\section{Author Contributions}

D.M.C. and Q.C. contributed equally to this work.

Notes

The authors declare no competing financial interest.

\section{ACKNOWLEDGMENTS}

We thank the UK EPSRC through Programme Grant EP/ M025330/1 Hybrid Polaritonics, the Max Planck Society, and the European Commission through the Graphene Flagship for funding this research. L.C.F. acknowledges support from the European Commission (project WASPS, 618078).

\section{REFERENCES}

(1) Novoselov, K. S.; Fal'ko, V. I.; Colombo, L.; Gellert, P. R.; Schwab, M. G.; Kim, K. Nature 2012, 490, 192-200.

(2) Ohta, T.; Bostwick, A.; Seyller, T.; Horn, K.; Rotenberg, E. Science 2006, 313, 951-954.

(3) Castro, E. V.; Novoselov, K. S.; Morozov, S. V.; Peres, N. M. R.; dos Santos, J. M. B. L.; Nilsson, J.; Guinea, F.; Geim, A. K.; Neto, A. H. C. Phys. Rev. Lett. 2007, 99, 216802.

(4) Balog, R.; et al. Nat. Mater. 2010, 9, 315-319.

(5) Nevius, M. S.; Conrad, M.; Wang, F.; Celis, A.; Nair, M. N.; Taleb-Ibrahimi, A.; Tejeda, A.; Conrad, E. H. Phys. Rev. Lett. 2015, $115,136802$.

(6) Zhang, Y.; Tang, T.-T.; Girit, C.; Hao, Z.; Martin, M. C.; Zettl, A.; Crommie, M. F.; Shen, Y. R.; Wang, F. Nature 2009, 459, 820823.

(7) Han, M. Y.; Özyilmaz, B.; Zhang, Y.; Kim, P. Phys. Rev. Lett. 2007, 98, 206805.

(8) Yan, X.; Li, B.; Li, L.-s. Acc. Chem. Res. 2013, 46, 2254-2262.

(9) Bacon, M.; Bradley, S. J.; Nann, T. Particle \& Particle Systems Characterization 2014, 31, 415-428.

(10) Narita, A.; Wang, X.-Y.; Feng, X.; Müllen, K. Chem. Soc. Rev. 2015, 44, 6616-6643.

(11) Wu, J.; Pisula, W.; Müllen, K. Chem. Rev. 2007, 107, 718-747.

(12) Tan, Y.-Z.; Yang, B.; Parvez, K.; Narita, A.; Osella, S.; Beljonne, D.; Feng, X.; Müllen, K. Nat. Commun. 2013, 4, 2646.

(13) Osella, S.; Narita, A.; Schwab, M. G.; Hernandez, Y.; Feng, X.; Müllen, K.; Beljonne, D. ACS Nano 2012, 6, 5539-5548.

(14) Chen, Z.; et al. J. Am. Chem. Soc. 2017, 139, 3635-3638.

(15) Kasprzak, J.; Richard, M.; Kundermann, S.; Baas, A.; Jeambrun, P.; Keeling, J. M. J.; Marchetti, F. M.; Szymanska, M. H.; Andre, R.; Staehli, J. L.; Savona, V.; Littlewood, P. B.; Deveaud, B.; Dang, L. S. Nature 2006, 443, 409.

(16) Schneider, C.; et al. Nature 2013, 497, 348-352.

(17) Amo, A.; Sanvitto, D.; Laussy, F. P.; Ballarini, D.; del Valle, E.; Martin, M. D.; Lemaitre, A.; Bloch, J.; Krizhanovskii, D. N.; Skolnick, M. S.; Tejedor, C.; Vina, L. Nature 2009, 457, 291.

(18) Antoine-Vincent, N.; Natali, F.; Byrne, D.; Vasson, A.; Disseix, P.; Leymarie, J.; Leroux, M.; Semond, F.; Massies, J. Phys. Rev. B: Condens. Matter Mater. Phys. 2003, 68, 153313.

(19) Weisbuch, C.; Nishioka, M.; Ishikawa, A.; Arakawa, Y. Phys. Rev. Lett. 1992, 69, 3314-3317.

(20) Liu, X.; Galfsky, T.; Sun, Z.; Xia, F.; Lin, E.-c.; Lee, Y. H.; KénaCohen, S.; Menon, V. M. Nat. Photonics 2014, 9, 30-34.
(21) Flatten, L. C.; He, Z.; Coles, D. M.; Trichet, A. A. P.; Powell, A. W.; Taylor, R. A.; Warner, J. H.; Smith, J. M. Sci. Rep. 2016, 6, 33134. (22) Lidzey, D. G.; Bradley, D. D. C.; Skolnick, M. S.; Virgili, T.; Walker, S.; Whittaker, D. M. Nature 1998, 395, 53-55.

(23) Hutchison, J. A.; Schwartz, T.; Genet, C.; Devaux, E.; Ebbesen, T. W. Angew. Chem., Int. Ed. 2012, 51, 1592-1596.

(24) Ebbesen, T. W. Acc. Chem. Res. 2016, 49, 2403-2412.

(25) Graf, A.; Tropf, L.; Zakharko, Y.; Zaumseil, J.; Gather, M. C. Nat. Commun. 2016, 7, 13078.

(26) Engel, M.; Steiner, M.; Lombardo, A.; Ferrari, A. C.; Löhneysen, H. v.; Avouris, P.; Krupke, R. Nat. Commun. 2012, 3, 906.

(27) Furchi, M.; Urich, A.; Pospischil, A.; Lilley, G.; Unterrainer, K.; Detz, H.; Klang, P.; Andrews, A. M.; Schrenk, W.; Strasser, G.; Mueller, T. Nano Lett. 2012, 12, 2773-2777.

(28) Paternò, G. M.; Chen, Q.; Wang, X.-Y.; Liu, J.; Motti, S. G.; Petrozza, A.; Feng, X.; Lanzani, G.; Müllen, K.; Narita, A.; Scotognella, F. Angew. Chem., Int. Ed. 2017, 56, 6753-6757.

(29) Li, J.-L.; Bao, H.-C.; Hou, X.-L.; Sun, L.; Wang, X.-G.; Gu, M. Angew. Chem., Int. Ed. 2012, 51, 1830-1834.

(30) Pan, D.; Guo, L.; Zhang, J.; Xi, C.; Xue, Q.; Huang, H.; Li, J.; Zhang, Z.; Yu, W.; Chen, Z.; Li, Z.; Wu, M. J. Mater. Chem. 2012, 22, 3314-3318.

(31) Tischler, J. R.; Bradley, M. S.; Bulović, V.; Song, J. H.; Nurmikko, A. Phys. Rev. Lett. 2005, 95, 036401.

(32) George, J.; Wang, S.; Chervy, T.; Canaguier-Durand, A.; Schaeffer, G.; Lehn, J.-M.; Hutchison, J. A.; Genet, C.; Ebbesen, T. W. Faraday Discuss. 2015, 178, 281-294.

(33) Coles, D. M.; Michetti, P.; Clark, C.; Tsoi, W. C.; Adawi, A. M.; Kim, J.-S.; Lidzey, D. G. Adv. Funct. Mater. 2011, 21, 3691-3696.

(34) Grant, R. T.; Michetti, P.; Musser, A. J.; Gregoire, P.; Virgili, T.; Vella, E.; Cavazzini, M.; Georgiou, K.; Galeotti, F.; Clark, C.; Clark, J.; Silva, C.; Lidzey, D. G. Adv. Opt. Mater. 2016, 4, 1615-1623.

(35) Cookson, T.; Georgiou, K.; Zasedatelev, A.; Grant, R. T.; Virgili, T.; Cavazzini, M.; Galeotti, F.; Clark, C.; Berloff, N. G.; Lidzey, D. G.; Lagoudakis, P. G. Adv. Opt. Mater. 2017, 1700203.

(36) Panzarini, G.; Andreani, L. C.; Armitage, A.; Baxter, D.; Skolnick, M. S.; Astratov, V. N.; Roberts, J. S.; Kavokin, A. V.; Vladimirova, M. R.; Kaliteevski, M. A. Phys. Rev. B: Condens. Matter Mater. Phys. 1999, 59, 5082-5089.

(37) Armitage, A.; Lidzey, D.; Bradley, D.; Virgili, T.; Skolnick, M.; Walker, S. Synth. Met. 2000, 111-112, 377-379.

(38) Bajoni, D. J. Phys. D: Appl. Phys. 2012, 45, 313001.

(39) Houdré, R. Phys. Status Solidi B 2005, 242, 2167-2196.

(40) Plumhof, J. D.; Stöferle, T.; Mai, L.; Scherf, U.; Mahrt, R. F. Nat. Mater. 2013, 13, 247-252.

(41) Daskalakis, K. S.; Maier, S. A.; Murray, R.; Kéna-Cohen, S. Nat. Mater. 2014, 13, 271-278.

(42) Litinskaya, M.; Reineker, P.; Agranovich, V. M. J. Lumin. 2004, $110,364-372$.

(43) Michetti, P.; La Rocca, G. C. Phys. Rev. B: Condens. Matter Mater. Phys. 2009, 79, 035325.

(44) Litinskaya, M.; Reineker, P.; Agranovich, V. M. J. Lumin. 2006, 119-120, 277-282.

(45) Michetti, P.; La Rocca, G. C. Phys. Rev. B: Condens. Matter Mater. Phys. 2008, 77, 195301.

(46) Somaschi, N.; Mouchliadis, L.; Coles, D.; Perakis, I.; Lidzey, D.; Lagoudakis, P.; Savvidis, P. Appl. Phys. Lett. 2011, 99, 143303.

(47) Grant, R. T.; Michetti, P.; Musser, A. J.; Gregoire, P.; Virgili, T.; Vella, E.; Cavazzini, M.; Georgiou, K.; Galeotti, F.; Clark, C.; Clark, J.; Silva, C.; Lidzey, D. G. Adv. Opt. Mater. 2016, 4, 1615-1623.

(48) Kéna-Cohen, S.; Forrest, S. Nat. Photonics 2010, 4, 371-375.

(49) Mazza, L.; Fontanesi, L.; La Rocca, G. C. Phys. Rev. B: Condens. Matter Mater. Phys. 2009, 80, 235314.

(50) Mazza, L.; Kéna-Cohen, S.; Michetti, P.; La Rocca, G. C. Phys. Rev. B: Condens. Matter Mater. Phys. 2013, 88, 075321. 